\title{
Dirac Structures on Banach Lie Algebroids
}

\author{
Vlad-Augustin VULCU
}

\begin{abstract}
In the original definition due to A. Weinstein and T. Courant a Dirac structure is a subbundle of the big tangent bundle $T M \oplus T^{*} M$ that is equal to its ortho-complement with respect to the so-called neutral metric on the big tangent bundle. In this paper, instead of the big tangent bundle we consider the vector bundle $E \oplus E^{*}$, where $\mathrm{E}$ is a Banach Lie algebroid and $E^{*}$ its dual. Recall that $E^{*}$ is not in general a Lie algebroid. We define a bilinear and symmetric form on the vector bundle $E \oplus E^{*}$ and say that a subbundle of it is a Dirac structure if it is equal with its orthocomplement. Our main result is that any Dirac structure that is closed with respect to a type of Courant bracket, endowed with a natural anchor is a Lie algebroid. In the proof the differential calculus on a Lie algebroid is essentially involved. We work in the category of Banach vector bundles.
\end{abstract}

\section{Introduction}

In [4] A. Weinstein and T. Courant and then T. Courant in [3], replacing certain linear maps by their graphs, propose the notion of Dirac structure as a subbundle of the big tangent bundle $T M \oplus T^{*} M$ that is equal to its orthocomplement with respect to the so-called neutral metric on the big tangent bundle.

The notion was afterwards intensively studied from the geometry point of view in connection with the generalized geometry ([11], [5] and the references

Key Words: Banach Lie algebroids, Dirac structures.

2010 Mathematics Subject Classification:

Received: July, 2013.

Revised: September, 2013.

Accepted: October, 2013. 
therein) and it was applied in the study of nonholonomic mechanical systems or mechanical systems with constraints ([7], [12] and the references therein).

A linear version of the Dirac structures defined for Hilbert or Banach spaces is used in the study of interconnected systems ([9] and the reference therein). This fact suggests to M. Anastasiei and A. Sandovici a study of the Dirac structures in the category of Banach vector bundles ([2]). They find conditions for a Dirac structure to be a Banach Lie algebroid. The notion of Banach Lie algebroid was introduced by M. Anastasiei, [1] and independently by F. Pelletier [10].

An algebraic version of the Dirac structures, independently on dimensions is done in the book [6] to the aim of applications to integrability of the evolution equations.

In this paper we continue the geometric study of the Dirac structures in the category of Banach vector bundles generalizing the main results from [2]. In Section 1 we recall the notion of Banach Lie algebroid and we develop a differential calculus on it. The Theorem 1.1 is proved. In Section 2, given a Banach Lie algebroid $E$ we introduce on $E \oplus E^{*}$ a symmetric bilinear form similar with the neutral metric on the big tangent bundle and define an almost Dirac structure as a subbundle of $E \oplus E^{*}$ that equals its ortho-complement. Then using the differential calculus on the Lie algebroid $E$ we construct a type of Courant bracket and we say that an almost Dirac structure is integrable or is a Dirac structure if it is closed with respect to it. Various characterizations of integrability are provided. One of it says that an integrable Dirac structure has a natural structure of Banach Lie algebroid. For general theory of Banach manifolds and Banach vector bundles we refer to [8].

\section{Banach Lie algebroids}

Let $M$ be a smooth i.e. $C^{\infty}$ Banach manifold modeled on a Banach space $\mathbb{M}$ and let $\pi: E \rightarrow M$ be a Banach vector bundle whose type fiber is a Banach space $\mathbb{E}$. We denote by $\tau: T M \rightarrow M$ the tangent bundle of $M$. Let $\Gamma(E)$ be the set of smooth sections in the vector bundle $(E, \pi, M)$ and $\mathcal{X}(M)$ the set of smooth sections in the tangent bundle of $M$ (vector fields on $M$ ). The both are $\mathcal{F}(M)$-modules, where $\mathcal{F}(M)$ is the ring of smooth real functions on $M$.

Definition 2.1. We say that the vector bundle $\pi: E \rightarrow M$ is an anchored vector bundle if there exists a vector bundle morphism $\rho: E \rightarrow T M$. The morphism $\rho$ will be called the anchor map.

Locally, $\rho$ reduces to a morphism $U \times \mathbb{E} \rightarrow U \times \mathbb{M},(x, v) \rightarrow\left(x, \rho_{U}(x) v\right)$ with $\rho_{U}(x) \in L(\mathbb{E}, \mathbb{M})$, the space of continuous linear maps from $\mathbb{E}$ to $\mathbb{M}$. We call $\rho_{U}(x)$ the l.r. of $\rho$. 
Example 2.2. The tangent bundle of $M$ is trivially anchored vector bundle with $\rho=I$ (identity).

Example 2.3. Let $A$ be a tensor field of type $(1,1)$ on $M$. It is regarded as a section of the bundle of linear mappings $L(T M, T M) \rightarrow M$ and also as a morphism $A: T M \rightarrow T M$. In other words, $A$ may be thought as an anchor map.

Example 2.4. Any subbundle of $T M$ is an anchored vector bundle with the anchor the inclusion map in TM.

Example 2.5. Let $\xi: F \rightarrow M$ be a submersion. The subspaces $V_{u} F=$ Ker $\xi_{*, u}$ of TF over $F$ denoted by VF form a subbundle called the vertical subbundle. By Example 1.3 this is an anchored Banach vector bundle.

The anchored vector bundles over the same base $M$ form a category. The objects are the pairs $\left(E, \rho_{E}\right)$ with $\rho_{E}$ the anchor of $E$ and a morphism $f$ : $\left(E, \rho_{E}\right) \rightarrow\left(F, \rho_{F}\right)$ is a vector bundle morphism $f: E \rightarrow F$ which verifies the condition $\rho_{F} \circ f=\rho_{E}$. Now we define a structure of Banach Lie algebroid.

Definition 2.6. Let $\pi: E \rightarrow M$ be a Banach vector bundle. We say that it has a Banach Lie algebroid structure if

1. It is an anchored vector bundle with the anchor $\rho_{E}: E \rightarrow T M$ and the induced morphism $\rho_{E}: \Gamma(E) \rightarrow X(M)$.

2. There exists defined a bracket $[,]_{E}$ on the space $\Gamma(E)$ that provides a structure of real Lie algebra on $\Gamma(E)$.

3. The following hold:

(i) $\rho:\left(\Gamma(E),[,]_{E}\right) \rightarrow(X(M),[]$,$) is a Lie algebra homomorphism and$

(ii) $\left[s_{1}, f s_{2}\right]_{E}=f\left[s_{1}, s_{2}\right]_{E}+\rho_{E}\left(s_{1}\right)(f) s_{2}$, for every $f \in \mathcal{F}(M)$ and $s_{1}, s_{2} \in \Gamma(E)$.

Example 2.7. The tangent bundle $\tau: T M \rightarrow M$ is a Banach Lie algebroid with the anchor the identity map and the usual Lie bracket of vector fields on $M$.

Example 2.8. For any submersion $\xi: F \rightarrow M$, the vertical bundle $V F$ over $F$ is an anchored Banach vector bundle. As the Lie bracket of two vertical vector fields is again a vertical vector field it follows that $\left(V F, i,[,]_{V F}\right)$, where $i: V F \rightarrow T F$ is the inclusion map, is a Banach Lie algebroid. This applies, in particular, to any Banach vector bundle $\pi: E \rightarrow M$. 
Let $\Lambda_{a}^{q}(E)$ be the vector bundle of alternating multilinear forms on E. The fiber at each point is the space $L_{a}^{q}\left(E_{x}\right)$ consisting of all $q$ - multilinear alternating continuous functions on the fiber $E_{x}, x \in M$. The sections $\Omega^{q}(E):=\Gamma\left(\Lambda_{a}^{q}(E)\right)$ will be called differential forms of degree q. The set $\Omega^{q}(E)$ is an $\mathcal{F}(M)$ - module. In particular, $\Omega^{q}(T M)$ will be denoted by $\Omega^{q}(M)$. The differential operator $d_{E}: \Omega^{q}(E) \rightarrow \Omega^{q+1}(E)$ is given by the formula

$$
\begin{aligned}
& \left(d_{E} \omega\right)\left(s_{0}, \ldots, s_{q}\right)=\sum_{i=0, \ldots, q}(-1)^{i} \rho_{E}\left(s_{i}\right) \omega\left(s_{0}, \ldots, \widehat{s}_{i}, \ldots, s_{q}\right) \\
& +\sum_{0 \leq i<j \leq q}(-1)^{i+j} \omega\left(\left[s_{i}, s_{j}\right]_{E}, s_{0}, \ldots \widehat{s}_{i}, \ldots, \widehat{s}_{j}, \ldots, s_{q}\right)
\end{aligned}
$$

for $s_{0}, s_{1}, \ldots, s_{q} \in \Gamma(E)$, where hat over a symbol means that symbol must be deleted.

It is well known that $d_{E}$ has the usual properties of the exterior differential operator. In the other words, if $\Omega(E)=\oplus_{q=0,1, \ldots} \Omega^{q}(E)$, where $\Omega^{0}(E)$ is identified to $\mathcal{F}(E)$, then $(\Omega(E), d)$ is a differential complex. Let $d_{q}$ be the restriction of $d$ to $\Omega^{q}(E)$. The property $d_{E}^{2}=0$ says that $I m d_{q-1} \subseteq \operatorname{Ker} d_{q}$ and so there arise the quotient spaces $H^{q}(E)=K e r d_{q} / I m d_{q-1}$ that are called the cohomology groups of the Lie algebroid $E$. If $\operatorname{Kerd}_{q}=\operatorname{Imd}_{q-1}, q=$ $0, \ldots, \infty$ then all cohomologies are trivial and the differential complex $(\Omega(E), d)$ is called exact.

We recall that the $\mathcal{F}(M)$ - module $\Gamma(E)$ has a Lie algebra structure provided by the bracket $[,]_{E}$.

Let $s$ be a section of $E$. We define the interior product( contraction) with respect to $s$ as a linear map $i_{s}: \Omega^{q}(E) \rightarrow \Omega^{q-1}(E)$, given by the formula

$$
i_{s}(\omega)\left(s_{1}, s_{2}, \ldots, s_{q-1}\right)=\omega\left(s, s_{1}, s_{2}, \ldots, s_{q-1}\right),
$$

if $q>1, i_{s} \omega=\omega(s)$ is $q=1$ and $i_{s} f=0$, for $\mathrm{f} \in \Omega^{0}(E)$. We extend the contraction with respect to a given section of $\Gamma(E)$ to a $\mathcal{F}(M)$-linear map $i: \Gamma(E) \rightarrow L\left(\Omega^{q}(E), \Omega^{q-1}(E)\right)$, given by $i(s)(\omega)=i_{s}(\omega)$, for $s \in \Gamma(E)$ and $\omega \in \Omega^{q}(E)$.

Theorem 1.1 The differential complex $\left(\Omega(E), d_{E}\right)$ is a complex over the Lie algebra $\left(\Gamma(E),[,]_{E}\right)$.

According to the definition of a complex over a Lie algebra (see p.11 in $[6]$ ), since we have already defined the operator $i$, it suffices to prove

Lemma 2.9. The linear operator $i$ has the following properties:

1. $i_{s} \circ i_{r}+i_{r} \circ i_{s}=0$, 
2. $i_{s}^{2}=0$,

3. $i_{s} \circ d_{E} \circ i_{r}+d_{E} \circ i_{s} \circ i_{r}=i_{r} \circ i_{s} \circ d_{E}+i_{r} \circ d_{E} \circ i_{s}+i_{[s, r]_{E}}$,

for any $s, r \in \Gamma(E)$.

Proof. The first two properties are equivalent and follow from the antisymmetry of the $q$-forms. As to the third property for $q=0$ there is nothing to prove due to the definition of the interior product. For $\omega \in \Omega^{1}(E)$ the third formula reduces to:

$$
\begin{aligned}
\left(d_{E}(\omega(r))\right)(s)= & d_{E} \omega(s, r)+\left(d_{E} \omega(s)\right)(r)+\omega\left([s, r]_{E}\right), \\
\left(d_{E}(\omega(r))\right)(s)= & \rho_{E}(s)(\omega(r))-\rho_{E}(r)(\omega(s)) \\
& -\omega\left([s, r]_{E}\right)+\rho_{E}(r)(\omega(s))+\omega\left([s, r]_{E}\right),
\end{aligned}
$$

which is clearly true. For a $q$-form $\omega$, the third formula follows by a direct computation based on the Lie algebroid properties of $E$.

Having defined the interior product with respect to a section of $E$ and the exterior differential of a $q$-form we may define the Lie derivative $L_{s}$ with respect to a section $s \in \Gamma(E)$ as follows:

1. $L_{s} f=\rho_{E}(s)(f)$, for $f \in \mathcal{F}(M)$,

2. $L_{s} r=[s, r]_{E}$, for $r \in \Gamma(E)$,

3. $L_{s} \omega=\left(d_{E} \circ i_{s}+i_{s} \circ d_{E}\right)(\omega)$, for $\omega \in \Omega^{q}(E), q=2,3, \ldots$.

For a $q$-form $\omega$, the Lie derivative $L_{s} \omega$ is explicitly given by:

$$
\left(L_{s} \omega\right)\left(s_{1}, \ldots s_{q}\right)=\rho_{E}(s)\left(\omega\left(s_{1}, \ldots s_{q}\right)\right)-\sum_{k=1}^{q} \omega\left(s_{1}, \ldots,\left[s, s_{k}\right]_{E}, \ldots, s_{q}\right) .
$$

Lemma 2.10. Let $L_{s}: \Omega^{q}(E) \rightarrow \Omega^{q}(E)$ be the Lie derivative with respect $s \in \Gamma(E)$ The following properties hold good:

1. $d_{E} \circ L_{s}=L_{s} \circ d_{E}$,

2. $i_{s} \circ L_{s}=L_{s} \circ i_{s}$,

3. $L_{[s, r]_{E}}=L_{s} \circ L_{r}-L_{r} \circ L_{s}$.

Proof. A direct checking by using (1.1). 


\section{Dirac Structures on $E \oplus E^{\star}$}

Let $E$ be a Banach Lie algebroid and $E^{\star}$ be its dual. We may consider the vector bundle $E \oplus E^{\star}$ over $M$. On $\Gamma\left(E \oplus E^{\star}\right)$ we consider the following bilinear map:

$$
\langle(s, \alpha),(v, \beta)\rangle_{+}=\frac{1}{2}\left(i_{v} \alpha+i_{s} \beta\right),
$$

for any $(s, \alpha),(v, \beta) \in \Gamma\left(E \oplus E^{\star}\right)$.

For a subbundle $\mathcal{D} \subset E \oplus E^{\star}$ we define its orthogonal complement $\mathcal{D}^{\perp}$, with respect to the symmetric bilinear map $\langle,\rangle_{+}$, by:

$$
\mathcal{D}^{\perp}=\left\{(v, \beta) \in \Gamma\left(E \oplus E^{\star} \mid\langle(s, \alpha),(v, \beta)\rangle_{+}=0 \text {, for all }(s, \alpha) \in \mathcal{D}\right\} .\right.
$$

On the sections of $E \oplus E^{\star}$ we define the Courant bracket:

$$
[(s, \alpha),(v, \beta)]_{C}=\left([s, v]_{E}, L_{s} \beta-L_{v} \alpha+\frac{1}{2} d_{E}\left(i_{v} \alpha-i_{s} \beta\right)\right),
$$

for any $(s, \alpha),(v, \beta) \in \Gamma\left(E \oplus E^{\star}\right)$. On the sections of $\mathcal{D}$ the Courant bracket becomes:

$$
[(s, \alpha),(v, \beta)]_{C}=\left([s, v]_{E}, L_{s} \beta-L_{v} \alpha+d_{E}(\alpha(v)),\right.
$$

for $(s, \alpha),(v, \beta) \in \Gamma(\mathcal{D})$.

Definition 3.1. An almost Dirac bundle or an almost Dirac structure on the Lie algebroid $E$ is a vector subbundle $\mathcal{D}$ of $E \oplus E^{\star} \rightarrow M$ such that

$$
\mathcal{D}=\mathcal{D}^{\perp} .
$$

Definition 3.2. We say that an almost Dirac bundle $\mathcal{D} \subset E \oplus E^{\star}$ is a a Dirac bundle or a Dirac structure or it is integrable if it is closed under the Courant bracket.

Example 3.3. $(E, 0)$ and $\left(0, E^{\star}\right)$ are Dirac structures.

For now, we are interested in determining equivalent conditions that a nontrivial almost Dirac structure $\mathcal{D} \subset E \oplus E^{\star}$ to be a Dirac structure. To this aim we evaluate the Jacobiator corresponding to $[,]_{C}$. This Jacobiator $J=\left(J_{1}, J_{2}\right): \Gamma\left(E \oplus E^{\star}\right)^{3} \rightarrow \Gamma\left(E \oplus E^{\star}\right)$ is given by:

$$
\begin{aligned}
\left(J_{1}, J_{2}\right)= & {\left[[(s, \alpha),(v, \beta)]_{C},(z, \gamma)\right]_{C}+} \\
+ & {\left[[(v, \beta),(z, \gamma)]_{C},(s, \alpha)\right]_{C} } \\
& +\left[[(z, \gamma),(s, \alpha)]_{C},(v, \beta)\right]_{C},
\end{aligned}
$$

for any $(s, \alpha),(v, \beta),(z, \gamma) \in \Gamma\left(E \oplus E^{\star}\right)$. Since $[,]_{E}$ is a Lie bracket $J_{1}$ vanishes. In general, $J_{2}$ does not vanish. 
Lemma 3.4. Let $\mathcal{D} \subset E \oplus E^{\star}$ be an almost Dirac Bundle. Then the second component $J_{2}$ of the Jacobiator corresponding to the Courant bracket $[,]_{C}$ is given by:

$$
\begin{aligned}
J_{2}= & \frac{1}{4} d_{E}\left(L_{s}(\beta(z)-\gamma(v))+L_{v}(\gamma(s)-\alpha(z))+L_{z}(\alpha(v)-\beta(s))\right) \\
& +\frac{1}{2} d_{E}\left(\gamma\left([s, v]_{E}\right)+\alpha\left([v, z]_{E}\right)+\beta([z, s])\right),
\end{aligned}
$$

for any $(s, \alpha),(v, \beta),(z, \gamma) \in \Gamma\left(E \oplus E^{\star}\right)$.

Furthermore, the restriction of $J_{2}$ to $\mathcal{D}$ is

$$
\begin{aligned}
J_{2}= & \frac{1}{2} d_{E}\left\{L_{s} \beta(z)+L_{v} \gamma(s)+L_{z} \alpha(v)\right\}+ \\
& +\frac{1}{2} d_{E}\left\{\gamma\left([s, v]_{E}\right)+\alpha\left([v, z]_{E}\right)+\beta\left([z, s]_{E}\right)\right\},
\end{aligned}
$$

for any $(s, \alpha),(v, \beta),(z, \gamma) \in \Gamma(\mathcal{D})$.

Proof. The first formula follows from the identities $L_{[s, v]_{E}}=L_{s} \circ L_{v}-L_{r} \circ L_{v}$ and $L_{s} \beta(z)=\rho_{E}(s)(\beta(z))-\beta\left([s, z]_{E}\right)$, while the second formula is a direct consequence of the first and of the equality $\mathcal{D}=\mathcal{D}^{\perp}$.

Definition 3.5. Let $\mathcal{D} \subset E \oplus E^{\star}$ be an almost Dirac bundle. On the sections of $\mathcal{D}$ we define a map

$$
T_{\mathcal{D}}((s, \alpha),(v, \beta),(z, \gamma))=\left\langle[(s, \alpha),(v, \beta)]_{C},(z, \gamma)\right\rangle_{+},
$$

for any $(s, \alpha),(v, \beta),(z, \gamma) \in \Gamma(\mathcal{D})$.

Lemma 3.6. Let $\mathcal{D} \subset E \oplus E^{\star}$ be an almost Dirac bundle. On the sections of $\mathcal{D}$ the map $T_{\mathcal{D}}$ satisfies the following identity:

$$
\begin{aligned}
T_{\mathcal{D}}((s, \alpha),(v, \beta),(z, \gamma))= & \alpha\left([v, z]_{E}\right)+\beta\left([z, s]_{E}\right)+\gamma\left([s, v]_{E}\right) \\
& +L_{s}(\beta(z))+L_{v}(\gamma(s))+L_{z}(\alpha(v)) \\
, \quad &
\end{aligned}
$$

for any $(s, \alpha),(v, \beta),(z, \gamma) \in \Gamma(\mathcal{D})$.

Proof. This formula follows from the definition of $\langle,\rangle_{+}$and the identity $\left(L_{u} \eta\right)(v)=\left(\rho_{E}(u)\right)(\eta(v))-\eta\left([u, v]_{E}\right)$.

Lemma 3.7. The second component $J_{2}$ of the Jacobiator of $[,]_{C}$, restricted to $\mathcal{D}$, satisfies the following identity:

$$
J_{2}((s, \alpha),(v, \beta),(z, \gamma))=\frac{1}{2} d_{E}\left(T_{\mathcal{D}}((s, \alpha),(v, \beta),(z, \gamma))\right),
$$

for any $(s, \alpha),(v, \beta),(z, \gamma) \in \Gamma(\mathcal{D})$. 
The formula from this Lemma is also a consequence of the identity used in the proof of Lemma 2.6. The same identity is used to check

Lemma 3.8. On the almost Dirac bundle $\mathcal{D} \subset E \oplus E^{\star}$ we have the following identity:

$$
\begin{aligned}
T_{\mathcal{D}}((s, \alpha),(v, \beta),(z, \gamma))= & -d_{E} \alpha(v, z)-d_{E} \beta(z, s)-d_{E} \gamma(s, v) \\
& +L_{s}(\gamma(v))+L_{v}(\alpha(z))+L_{z}(\beta(s)) \\
= & \left(L_{s} \beta\right)(z)+\left(L_{v} \gamma\right)(s)+\left(L_{z} \alpha\right)(v) .
\end{aligned}
$$

By the definition the map $T_{\mathcal{D}}$ is linear in the third argument. By Lemma 2.8 it is linear and totally-symmetric.

Now we prove the following characterizations of the integrability of an almost Dirac structure.

Proposition 3.9. Assume that $E$ is a Banach Lie algebroid and that $\mathcal{D}$ is an almost Banach Dirac structure on $E$. Then, $\mathcal{D}$ is integrable if and only if $T_{\mathcal{D}}=0$.

Proof. Since $\mathcal{D}$ is integrable, then for $(s, \alpha),(v, \beta) \in \Gamma(\mathcal{D})$ we have $[(s, \alpha),(v, \beta)]_{C} \in \Gamma(\mathcal{D})$ and so $T_{\mathcal{D}}=0$. Conversely, if $T_{\mathcal{D}}=0$ it follows that $[(s, \alpha),(v, \beta)]_{C} \in \Gamma\left(\mathcal{D}^{\perp}\right)=\Gamma(\mathcal{D})$. Thus $\mathcal{D}$ is integrable. This completes the proof.

Corollary 3.10. Assume that $E$ is a Banach Lie algebroid and that $\mathcal{D}$ is an almost Banach Dirac structure on $E$. Then, $\mathcal{D}$ is integrable if and only if

$$
\left(L_{s} \beta\right)(z)+\left(L_{v} \gamma\right)(s)+\left(L_{z} \alpha\right)(v)=0
$$

for all $(s, \alpha),(v, \beta),(z, \gamma) \in \Gamma(\mathcal{D})$.

Proof. It follows by a combination of the Lemma 2.8 and the Proposition 2.9 .

Theorem 3.11. Let $E$ be a Banach Lie algebroid and $\mathcal{D} \subset E \oplus E^{\star}$ be an almost Dirac bundle. Then $\mathcal{D}$ is a Dirac bundle if and only if $\mathcal{D}$ can be structured as a Lie algebroid.

Proof. Let $\mathcal{D} \subset E \oplus E^{\star}$ be a Dirac bundle. The Courant bracket furnishes a Lie algebra bracket [,] on the sections of $\mathcal{D}$ since $T_{\mathcal{D}}=0$ implies $J_{2}=0$.

Now we consider a vector bundle map $\sigma: E \oplus E^{\star} \rightarrow T M$, given by

$$
\sigma((s, \alpha))=\left(\rho_{E} \circ \rho\right)(s, \alpha)
$$

where $\rho$ is the canonical projection of $E \oplus E^{\star}$ onto $E$, i. e. $\rho((s, \alpha))=(s, 0)$, for $(s, \alpha) \in E \oplus E^{\star}$, and $\rho_{E}$ is the anchor of $E$. By a direct computation it 
comes out that the restriction of $\sigma$ to $\mathcal{D}$ acts as a derivation on the sections of $\mathcal{D}$, with respect to the Courant bracket.

Again a direct computation shows that $\sigma$ is a Lie algebra homomorphism.

Conversely, let $\left(\mathcal{D},[,]_{C}, \sigma\right) \subset E \oplus E^{\star}$ be an almost Dirac bundle that is structured as a Lie algebroid with respect to $[,]_{C}$ and to the map $\sigma$, previously defined. Then the Courant bracket [, $]_{C}$ restricted to $\mathcal{D}$ satisfies a Jacobi type identity i.e. $J_{2}=0$ and by the Lemma 2.7 and the linearity of $T_{\mathcal{D}}$ it follows $T_{\mathcal{D}}=0$. Thus by the Proposition $2.9, \mathcal{D}$ is a Dirac bundle.

Example 3.12. Let $\omega$ be a nondegenerate 2-form in $E$. It defines a map $\omega: E \rightarrow E^{*}$ by $e \rightarrow \omega(e): \Gamma(E) \rightarrow \mathbb{R}$ with $\omega(e) f=\omega(e, f), e, f \in \Gamma(E)$. We take $\mathcal{L}=$ graph $\omega=\left\{(e, \sigma) \mid e \in \Gamma(E), \sigma \in \Gamma\left(E^{*}\right)\right.$ with $\left.\sigma=\omega(e)\right\}$. Since $\mathcal{L}$ is a vector subbundle it defines a almost Dirac bundle. It is a Dirac bundle if and only if $\omega$ is closed with respect to $d_{E}$.

Acknowledgements The author was partially supported by a grant of the AMPOSDRU and the University "Al. I Cuza", of Iaşi, project number POSDRU/CPP 107/DMI 1.5/S/78342.

\section{References}

[1] M., Anastasiei, Banach Lie Algebroids. An. St. Univ. "Al.I. Cuza" Iasi S.N. Matematica, T. LVII, 2011 f.2, 409-416.

[2] M. Anastasiei, A. Sandovici, Banach Dirac bundles. Int. J. Geom. Methods Mod. Phys. 10(7), (2013), 1350033 (16 pages) DOI: 10.1142/S0219887813500333.

[3] T. Courant, Dirac structures, Trans. A.M.S. 319 (1990), 631-661.

[4] T. Courant, A. Weinstein, Beyond Poisson structures, Seminaire SudRhodanien de Geometrie, Travaux en cours 27 (1988), 39-49, Hermann, Paris.

[5] M. Crasmareanu, Dirac structures from Lie integrability, Int. J. Geom. Methods Mod. Phys., 9(4), (2012), 1220005,(6pages), DOI: 10.1142/S0219887812200058.

[6] I. Dorfman, Dirac structures and integrability of nonlinear evolution equations, John Wiley Sons, 1993.

[7] M. Jotz, T. S. Ratiu, Dirac structures, nonholonomic systems and reduction, Rep. Math. Phys, 69(1), 2012, 5-56. 
[8] S. Lang, Fundamentals of Differential Geometry, Graduate Text in Mathematics 191, Springer-Verlag New York, 1999.

[9] O. Iftime, A. Sandovici, "Interconnection of Dirac structures via kernel/image representation", In Proceedings of the American Control Conference, pp. 3571-3576, June 29-July 1, 2011.

[10] F. Pelletier, Integrability of weak distributions on Banach manifolds, Indag. Math. (N.S.) 23(2012), no. 3, 214-242.

[11] I. Vaisman, Dirac structures on generalized Riemannian manifolds, Rev. Roum. Math. Pures Appl., 17(2), 2012, 179-203.

[12] H. Yoshimura, H. Jacobs, J. E. Marsden, Interconnection of Dirac Structures and Lagrange-Dirac Dynamical Systems, In Proceedings of the $19^{\text {th }}$ International Symposium on Mathematical Theory of Networks and Systems, Budapest, Hungary, pp. 5-9, July 2010.

Vlad-Augustin Vulcu,

Faculty of Mathematics,

"Al. I. Cuza" University,

Bd. Carol I, Iasi, 700508, Romania,

Email: vulcuvlad@gmail.com 